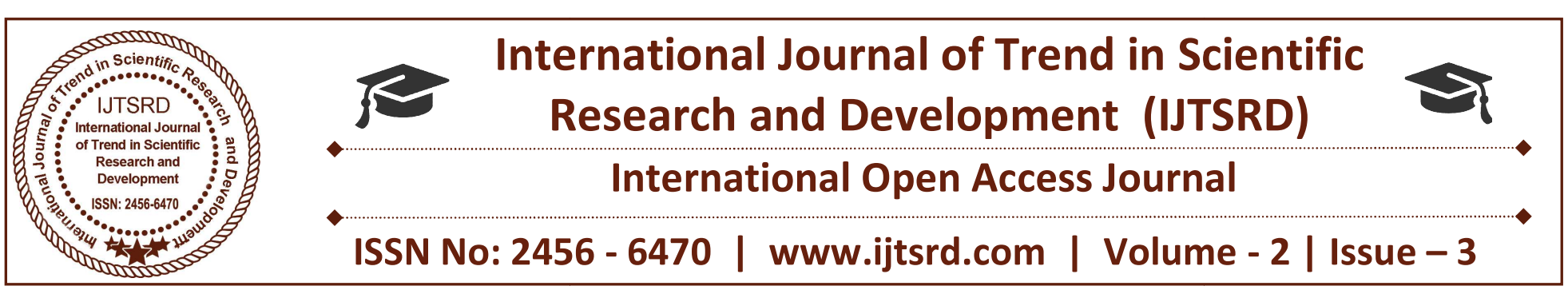

\title{
Design of a Real Time Modular Brain-Computer Interface movement-related EEG recognition
}

\author{
R. Nishanth, Naveen. S, Abin John Joseph \\ Assistant Professor, Cochin University College of Engineering Kuttanad, Pulincunnu, Kerala, India
}

\begin{abstract}
We have been managing an exploration in the field of development related EEG acknowledgment and right now we are taking a shot at a disconnected single trial order framework ready to perceive development related EEG on the base of its transient advancement. This commitment displays a preparatory investigation of the proposed continuous BCI framework engineering parts for the EEG Processing Pipeline alongside the aftereffects of the outline of the principal essential framework pieces. The venture likewise effectively includes understudies of our specialization in a genuine cooperation.
\end{abstract}

\section{INTRODUCTION}

Existing Brain Computer Interface (BCI) models experience the ill effects of too moderate correspondence channel between a human mind and a PC working with Information Transfer Rate (ITR) lower than 100 bits for each moment. One plausibility prompting higher ITR is the acknowledgment of more unmistakable mind states - exchanging more bits per state - high-determination EEG acknowledgment. Be that as it may, existing BCI frameworks perceive just couple of altogether different EEG exercises (left/right-hand or finger development, mental exercises, cognizant EEG mood control, or occasion related possibilities, among others). Our examination is focused to the investigation of potential outcomes of the high-determination development acknowledgment from the EEG signal. The Hidden Markov Models (HMMs) classifier utilized EEG worldly progression, instead of data put away in the distinction of flag powers from various terminals

generally utilized as a part of frameworks eg perceiving left and right hand developments, feet and tongue developments, or mental exercises. Right now our exertion is coordinated to enhance the entire calculation initially to have the capacity to process ceaseless EEG stream (not just single trials) and after that to work continuously. Proposed continuous application permits utilizing visual input which is known to authorize the development related cerebrum designs [2] as the development arranging, execution and perception systems are interconnected. This can additionally expand arrangement exactness. To achieve the arranged outcomes in a plausible time we began three parallel ventures:

1. Research in the field of visually impaired source partition application on EEG to additionally increment achieved order score with the Single-trial disconnected grouping framework.

2. Research in the field of nonstop EEG preparing focused to augmentation of ebb and flow characterization calculations to a consistently working framework.

3. Design of continuous EEG Processing Pipeline (EPP) programming.

The third task was additionally separated into two stages:

1. In the primary stage the objective is to completely configurations, confirm, and assess essential EPP and after that utilization it for straightforward assignment of left/right hand 
development acknowledgment with visual input hand preceding onward a PC screen.

2. In the second stage the EPP pipeline will be moved up to execute the constant EEG acknowledgment calculations we are right now dealing with in the casing of the second task. This commitment introduces a preparatory investigation of the proposed continuous BCI framework compositional segments for the EEG Processing Pipeline (EPP, see Fig. 1) and compresses the primary outcomes from the stage 1 of the outline.
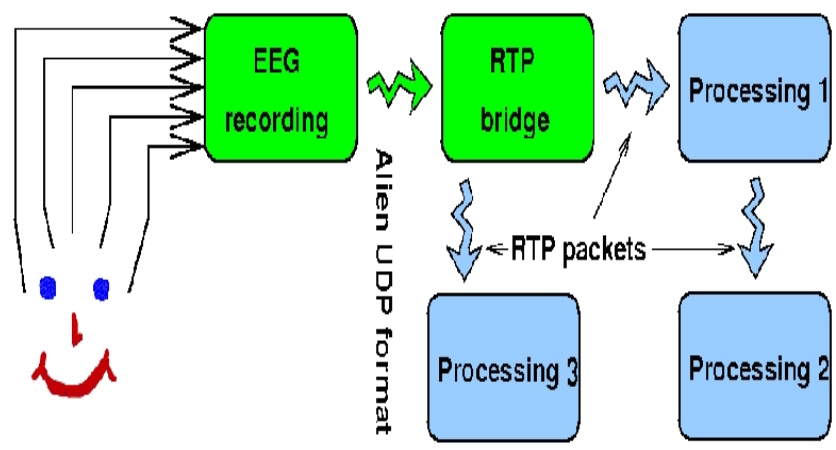

Figure 1: Modular architecture of the EEG Processing Pipeline (EPP).

\section{EPP ARCHITECTURE}

Since the EPP BCI calculations have been still a work in progress, we chose to manufacture the entire EPP of inexactly coupled pieces associated by means of system parcel interfaces. This enables us to effortlessly supplant usage of parts of the framework alongside future advancement of new flag handling calculations. EEG machine (producer Alien Technik) outfitted with a system interface gushing the EEG by means of UDP parcels in an exclusive Alien configuration was utilized for tests. Be that as it may, we needed to be autonomous on the utilized EEG machine, consequently the principal obstruct in our EPP is an extension changing over Alien UDP bundles into our system EEG design, see Fig. 1. module correspondence convention. Its focal points are datagram nature (important to keep low inactivity) and prioritization in organize switches over other noncontinuous information streams. In spite of the fact that RTP is much of the time utilized for media transmission in the field of telemedicine [3, 4], we don't know about any utilization of this convention in the field of EEG flag handling; lion's share of papers writing about telemedicine EEG preparing bargain just with disconnected transmission of EEG information. Since the first RTP convention does not specifically bolster biomedical (EEG) information exchange, we built up an expansion to the convention, see $[5,6]$. For the itemized depiction of EPP EEG RTP bundle header, see [5]. The expansion was made in a path not to meddle with ordinary RTP activity so the EEG bundles can be prepared by any product or equipment effectively supporting RTP information streams. Java was decided for the framework usage because of its multiplatformness. As the transfer speed prerequisites for crude EEG information transmission are just direct, Java usage ought to be sufficiently quick. An accessible open-source RTP usage [7] was utilized to execute the RTP stack.

\section{DESIGN OF THE EPP}

The plan of the EPP nearly mirrors the genuine BCI EEG preparing pipeline attracted Figure 3. Each square in the $\mathrm{BCI}$ structure is spoken to by one module in the planned programming. The modules are associated by organize bundle interfaces and are actualized utilizing multithreading and parallelized approach to abuse however much as could be expected the equipment parallelism offered by the present multicore and hyperthreading CPUs. As of now the EEG machine interface module, RTP connect, RTP recipient, and information stream watcher modules are actualized. Highlight extraction, surface sifting, and grouping modules are being worked on.

\section{SURFACE FILTERING}

There are a couple of layers of head tissue in the middle of the cerebrum dipole sources and scalp cathodes: cerebrospinal liquid, head tissues, skull bone, and scalp bringing about low-recurrence band pass spatial sifting of the EEG movement. Because of this the scalp possibilities are obscured and any very restricted EEG movement turns out to be less clear. This is in logical inconsistency with the need of mind PC interface tests requiring EEG action with as low spatial obscuring as could reasonably be expected. The surface channel repays this obscuring to some degree. The surface sifting module executes a discrete Laplacian channel [8]. The discrete Laplacian channel was picked as it has some surprising favorable circumstances - it requires just a low computational power, it is effectively relevant (straightforward direct mix of the cathode possibilities), and gives rather great outcomes BCI tests. 


\section{FEATURE EXTRACTION}

The point of the present period of the undertaking is to build up an ongoing working BCI EPP perceiving left and hand development from the EEG flag. To achieve this undertaking, only a basic parameterization calculation is required. The element extraction module needs to register controls in predefined subbands of the EEG flag so as we may distinguish the $\mu$ mood constriction going with the development [9]. FIR channels will be utilized to sift through subbands of the EEG $\mu$ band and flag controls in the subbands will be utilized as highlights.

\section{DATA FLOW MONITORING}

Observing of the EEG information streams is vital for framework troubleshooting and additionally for the framework activity checking. A devoted perception module was executed to imagine the between piece organize RTP activity. The screen has the accompanying highlights:

All kinds of dataflow (crude EEG, surface separated EEG, extricated highlights)/can be imagined "on the oscilloscope", see Fig. 2.

$>$ Missing parcels (lost eg because of system clog) are set apart with red boxes,

$>$ Special labels (antiquity, development, and so on) are shown in the "degree" show.

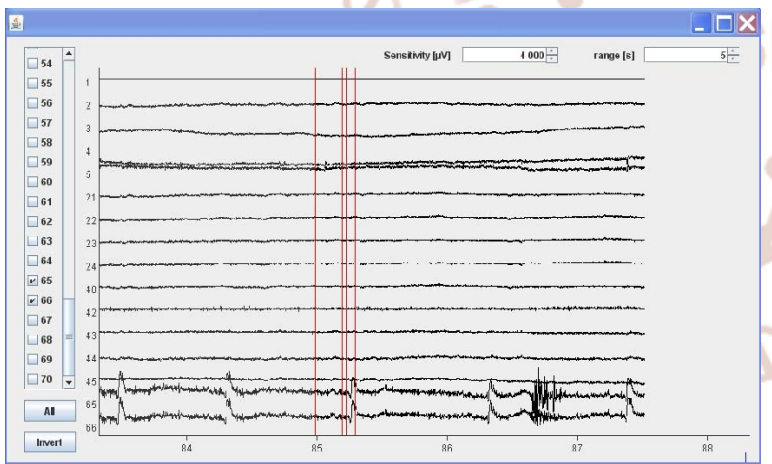

Figure 2: EPP data flow monitoring utility screenshot during raw EEG processing. The red lines indicate missing packets (data packets which were lost during transmission).

\section{CLASSIFIER}

The assignment of left/right hand development acknowledgment needs just a straightforward classifier. The standard Perceptron is required to give adequate execution in this framework. The framework comfort will be utilized to switch amongst preparing and testing methods of the framework. The module will control the preparation as per a predefined session convention.

\section{VERIFICATION}

The designed system has been extensively tested. The following approaches have been used for system verification:

\section{Testing with the real EEG machine:}

done irregularly as the EEG machine is in a remote location.

System verification with simulated EEG

Machine: done regularly after each major change to the system. A set of testing scripts was prepared [6] to support regression testing of the basic functionalities. A dedicated Alien packet generator tool was developed so as we may test the whole EPP off-line, without access to the EEG machine. Alien packet generator compliance with the real device is verified with the help of utility [10].

EEG data recorded in the frame of experiment [9] are used for testing with simulated EEG machine.

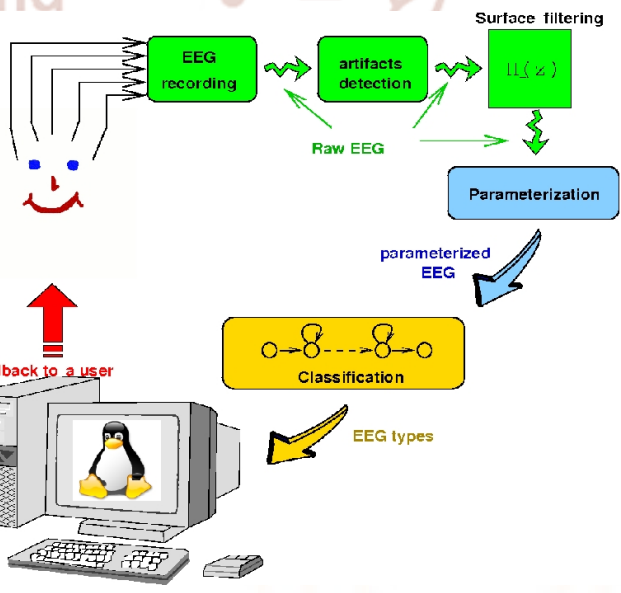

Figure 3: A simplified diagram of a BCI system

\section{SYSTEM PERFORMANCE}

The greatest worry amid programming advancement was a low inactivity without bounds EPP. The inactivity of the entire EPP is estimated as time between the example getting by the EEG machine and its handling and refreshing the data on the client's screen. The general framework idleness is an entirety of the accompanying parts: 
$>$ Packed age inertness is caused when quantization. In the event that one bundle contains eg $20 \mathrm{~ms}$ of information, at that point the postponement between the examining of the principal EEG test in the parcel and bundle age by the EEG machine is $20 \mathrm{~ms}$. This deferral is unavoidable and can be diminished by decreasing parcel measure.

$>$ Network idleness is the vehicle deferral of the neighborhood. Creators of work [11] utilizes UDP convention to transmit one channel EEG more than $200 \mathrm{~km}$ arrange interface keeping in mind the end goal to keep up dosing of analgesic medication. The framework worked with $1 \mathrm{sec}$ parcel measure, transport idleness was of approx. $8 \mathrm{~ms}$. The system inactivity ought to be limited by utilizing the RTP convention which is organized in arrange gear. Every correspondence connect between two modules add this inactivity to the general framework inertness.

$>$ Packed preparing dormancy is the idleness presented by every one of the modules in the framework. It can be lessened by a watchful written work and profiling/enhancement of our product. Every module includes its own particular segment of this dormancy to the general framework idleness. For constant control, idleness should be beneath $60 \mathrm{~ms}$ [12]. We gauged inertness and throughput of the framework with the at present planned modules. The primary stable arrival of the framework (Alien generator, RTP connect, RTP collector) had an inactivity of $17 \mathrm{~ms}$ which was at that point enough for constant control. Additionally profiling demonstrated that regular dynamic memory designations were backing off the entire framework. Subsequent to improving every one of the parts of the framework we accomplished almost zero idleness and immaterial parcel misfortune rate over a neighborhood. The officially actualized piece of the framework is skilled to function as quick as 20 times the continuous speed in reproduced tests without critical bundle misfortune. The outcomes demonstrate that - despite the fact that Java is typically considered as a moderate dialect precisely composed multi-threading applications can extremely meet delicate ongoing limitations and in this manner there is no compelling reason to utilize Real Time Java augmentation [13] for our situation. We additionally assessed the transmission capacity expected to exchange the EEG motion all through the system joins. In the event that we have to transmit 45 channel EEG at examining rate of $256 \mathrm{~Hz}, 16$ bit for every example, we require only $45 \times 256 \times 2 \mathrm{~B} / \mathrm{sec}=$ $22,5 \mathrm{kB} / \mathrm{sec}=180 \mathrm{~kb} / \mathrm{sec}$ which is just a minor division of limit offered by standard $100 \mathrm{Mb}$ Ethernet organize connect. The outcomes show that no EEG information pressure for transmission is required.

\section{CONCLUSIONS}

An establishment for a BCI secluded framework configuration was executed. The modules convey through an all around characterized interface which is free on the genuine EEG recording machine. The test comes about demonstrated that the achieved inertness is attainable and Java usage can deal with information stream. The proposed particular engineering permits to unreservedly disperse parts of the BCI EPP crosswise over system; while eg the element extraction and example acknowledgment can dwell on a capable PC, the last introduction layer can keep running on any thin customer (eg cell phone, surrounding light controller, entryways opening, and so forth) generously broadening the span of action of conceivable BCI gadget. The proposed disseminated engineering additionally permits to effortlessly misuse parallelism offered by the present multicore frameworks; the EPP modules can be basically keep running on various CPUs. Utilizing the standard RTP convention guarantees constant preparing of EEG information in any neighborhood. Last, yet not slightest, working with the group gives understudies an interesting background to partake in genuine cooperation; all the synergistic applications and philosophy are completely required for our task as every one of the members have been working from their homes and the venture members are subsequently greatly disseminated. The most recent stable arrival of all the created bundles is openly accessible at our site [5], forthcoming clients are compassionately requested to refer to this article.

\section{REFERENCES}

1) J. Doležal, J. Štastný, P. Sovka. Recognition of Direction of Finger Movement From EEG Signal Using Markov Models. In: 3rd European Medical and Biological Conference on Biomedical Engineering. EMBEC 2005, Prague, pp. 336-341

2) Christa Neuper. Feedback-Regulated Mental Imagery in BCI Applications: Using NonInvasive EEG and NIRS Signals. BBCI 
Workshop 2009 - Advancesin

Neurotechnologies. Berlin, 2009

3) Ilias Maglogiannis, Constantinos Delakouridis, Leonidas Kazatzopoulos. Enabling Collaborative Medical Diagnosis Over the Internet via Peer-toPeer Distribution of Electronic Health Records. J Med Syst, 2006, 30, 107-116

4) J. C. Guerri et al. A multimedia telemedicine system to assess musculoskeletal disorders. Proceedings of the 2003 International Conference on Multimedia and Expo - Volume 2. Pages: 701 704 Year of Publication: 2003 ISBN:0-78037965-9

5) Real Time BCI Project Page. http://amber.feld.cvut.cz/fpga/projects/ real_time_bci.html

6) Kubový J. Real-time EEG network transmission, Bachelor Thesis CTU FEE, Department of circuit theory, FPGA Laboratory, June 2009 (in Czech)

7) LibRTP hibrary. h:/ sourceforge.net/projects/jlibrtp/

8) Dennis J. McFarland, Lynn M. McCane, Stephen V. David, and Jonathan R. Wolpaw. Spatial filter selection for EEG-based communication. Electroencephalography and Relinical Neurophysiology, (103):386-394, 1997.

9) Doležal J., Štastný J., Sovka P., Recording and recognition of movement related EEG signal. In Applied Electronics, Applied Electronics, pp 9598, September 2009. ISBN 978-80-7043-781-0

10) UDP receiver and personal communication with Alien Technik

11) H. Ihmsen, K. Naguib, G. Schneider, H. Schwilden, J. Schüttler and E. Kochs. Teletherapeutic drug administration by long distance closed-loop control of propofol. British Journal of Anaesthesia, January 11, 2007, doi:10.1093/bja/ael337 